\title{
Isolation, Identification and Antibiotic Susceptibility Testing of Pseudomonas aeruginosa and Acinetobacter baumannii from Endotracheal Secretions in a Tertiary Care Centre
}

\author{
Seba Miriam David ${ }^{1}$, Chithra Jayaprakash ${ }^{2 *}$ and Anna Mathew ${ }^{3}$ \\ ${ }^{1}$ Interne MOSC Medical College, Kolenchery, Kerala-682311, India \\ ${ }^{2}$ Department of Microbiology, MOSC Medical College, Kolenchery, \\ Kerala, 682311, India \\ ${ }^{3}$ Department of Pharmacology, MOSC Medical College, Kolenchery, Kerala, 682311, India \\ *Corresponding author
}

\section{Ke ywords \\ Pseudomonas aeruginosa, Acinetobacterbaum anii, Non- ferementing bacilli, Antibiotic susceptibility, Tigecycline, Colistin}

Article Info

Accepted:

08 January 2020

Available Online:

10 February 2020
A B S T R A C T

Pseudomonas aeruginosa and Acinetobacter baumanii are aerobic non fermenting gram negative opportunistic pathogens that are major causes of nosocomial infections. Both these microorganisms thrive well in nutrient-deprived conditions and are responsible for a wide spectrum of infections. They show a higher predilection for infection in patients with diminished host defenses. Intensive care units are considered the epicenter of antibiotic resistant infections. Outbreaks are mainly associated with invasive procedures, cross infection via the hands of health staff and prolonged use of broad spectrum antibiotics. The rapidly spreading nature of carbapenem resistance among these organisms has raised concern over the reliability of carbapenems in managing critically ill patients. Successful management of multidrug resistant infections depends on strict compliance to infection control measures and judicious antibiotic use. The main aim of this study includes to isolate and identify Pseudomonas aeruginosa and Acinetobacter baumannii from endotracheal secretions and perform their antibiotic susceptibility test. Also to establish the prevalence of these organisms in ventilated patients in intensive care units. Approval was obtained from the ethics committee and waiver of informed consent was sought. The samples for this cross-sectional study were endotracheal secretions from patients, processed using standard microbiological procedures and antibiotic susceptibility testing done using Kirby-Bauer disc diffusion method. After incubation the results were read and interpreted as per CLSI guidelines. Non-fermenters constituted $60.24 \%$ of the isolates recovered from the 83 endotracheal secretion samples. Pseudomonas aeruginosa was the most common non- fermenter isolated (54\%), followed by Acinetobacter baumanii (30\%). Pseudomonas aeruginosa was the most commonly isolated non-fermenter (54\%) followed by Acinetobacter baumannii(30\%). From our study, aminoglycosides like amikacin and gentamicin were found to have good efficacy against Pseudomonas aeruginosa. Acinetobacter baumannii remained highly resistant to most of the antibiotics tested except colistin and tigecycline. 


\section{Introduction}

Pseudomonas aeruginosa (P.aeruginosa) and Acinetobacter baumannii (A.baumannii) are aerobic non-fermenting gram negative opportunistic pathogens that are major causes of nosocomial infections. Both these microorganisms thrive well in nutrientdeprived conditions and are responsible for a wide spectrum of infections including pneumonia, urinary tract infections, wound infections, meningitis and septicemia ${ }^{[1]}$. They show a higher predilection for infection in patients with diminished host defenses. Intensive care units are considered the epicenter of antibiotic resistant infections. Outbreaks are mainly associated with invasive procedures, cross infection via the hands of health staff and prolonged use of broad spectrum antibiotics ${ }^{[1,2]}$.

In intubated patients, the endotracheal tube gets readily colonized by bacteria within 24 hours. Biofilm formation and adherence using pili will aid their survival ${ }^{[3,4]}$. These organisms express a variety of determinants that confer resistance to several antibiotics ${ }^{[5]}$. Earlier studies have shown a higher in-patient mortality rate with infections due to Carbapenem-Resistant Acinetobacter baumannii (CRAB). The rapidly spreading nature of carbapenem resistance among these organisms has raised concern over the reliability of carbapenems in managing critically ill patients. Limited treatment options against these multidrug resistant strains has led to the revival of an old antibiotic colistin, which was not in use for many years owing to its properties of nephrotoxicity and neurotoxicity ${ }^{[6]}$. Successful management of multidrug resistant infections depends on strict compliance to infection control measures and judicious antibiotic use. This study therefore aims to evaluate the prevalence of these two organisms in the endotracheal secretions of patients admitted at this centre to appraise clinicians of their current antibiotic susceptibility and resistance patterns. It is intended to explore the potential of employing adequate empirical therapy which may save human lives and financial resources.

The main aim of this study includes to isolate and identify Pseudomonas aeruginosa and Acinetobacter baumannii from endotracheal secretions and perform their antibiotic susceptibility test. Also to establish the prevalence of these organisms in ventilated patients in intensive care units.

\section{Materials and Methods}

This study is a cross-sectional study on $P$. aeruginosa and A. baumannii obtained from endotracheal secretions from patients of this tertiary care hospital. This cross sectional study was conducted in the Microbiology department of this Medical college during the period April to June 2017.

Pseudomonas aeruginosa and Acinetobacter baumannii are non-fermenting gram negative organisms causing health care associated infections with evidence of increased resistance to antibiotics. An organism is considered a non-fermenter, if it fails to produce acid in routine glucose medium or utilizes glucose oxidatively. ${ }^{[7]}$

Isolation and identification of Non-fermenting Gram-negative Bacilli was done either as a pure growth in culture or as the predominant isolate in a mixed culture. Growth on triple sugar iron agar slant with no growth extending into the butt and no acidification of the slant was taken as that of a non-fermenter. Identification was done by studying the odour, colony morphology, pigment production, Gram staining, motility, biochemical reactions and antibiotic susceptibility test results. The culture media 
used to study the colony characteristics were Blood agar, Chocolate agar and MacConkey agar. The biochemical reactions used were oxidase test, indole production, Triple sugar iron agar, mannitol motility medium, urea hydrolysis, citrate utilization, nitrate reduction, and $10 \%$ lactose broth. ${ }^{[8]}$

Antibiotic susceptibility testing was done using the Kirby-Bauer disc diffusion method to study antibiotic susceptibility. A lawn culture of the bacterial suspension was made by spreading the entire surface of MuellerHinton agar using a sterile cotton swab dipped in the suspension. After five to ten minutes, filter paper discs impregnated with antibiotic of definite concentrations were placed on the surface of the medium using sterile forceps. After incubation at $37^{\circ} \mathrm{C}$ for 18 to 24 hours, the results were read by measuring the zone diameter in millimetres using a ruler and interpreted using standard interpretative charts as per CLSI (Clinical and Laboratory Standards Institute) guidelines ${ }^{[9]}$.

An antibiogram is the representation of the antibiotic sensitivity testing, where the susceptibility pattern of an isolated bacterial strain to different antibiotics is indicated.

\section{Sampling}

Consecutive sampling by serial recruitment of all endotracheal secretions coming to the laboratory, if selection criteria were fulfilled.

\section{Inclusion criteria}

All endotracheal secretions coming to the microbiology department were consecutively included in the study.

\section{Exclusion criteria}

The samples excluded were the improperly collected samples and repeat samples from the same patient already included in study.

\section{Ethical issues}

Institutional scientific review board and ethics committee approval along with the waiver of informed consent were obtained. Permission to conduct the study was obtained from the Medical Superintendent of this institution. All data were stored anonymously and handled only by the investigator and authorised personnel.

\section{Sample size}

Sample size was calculated using Master Sample Size computer software. ${ }^{[10]}$ In the study by Kalidas Rit et al., from Kolkata, out of 4180 clinical samples studied, 504 (12.05\%) non-fermenters (P.aeruginosa and A.baumannii) were isolated ${ }^{[2]}$. The proportion of 0.1205 was used to calculate the sample size for single proportion for a confidence interval of $95 \%$ and precision of $10 \%$.

\section{Study procedure}

Endotracheal secretions that reach the Microbiology laboratory of this institution were selected for the study. The samples were processed as soon as possible after collection. Endotracheal secretions were vortexed for one minute, centrifuged at $3000 \mathrm{rpm}$ for 10 minutes and semi-quantitative culture done using a calibrated inoculating loop which holds $0.001 \mathrm{ml}$ of the sample. The culture media used for inoculation were blood agar, chocolate agar and MacConkey agar. The inoculated plates were incubated at $37^{\circ} \mathrm{C}$ for 18-24 hours. Identification of the isolates were done by standard microbiological procedures such as colony morphology, Gram stain reactions and biochemical reactions mentioned above. Antibiotic susceptibility testing was performed by Kirby-Bauer disc diffusion method on Mueller-Hinton agar. After incubation at $37^{\circ} \mathrm{C}$ for $18-24$ hours, the results were read and interpreted as per CLSI guidelines. ${ }^{[9]}$ 


\section{Data analysis}

The data was entered in an Excel spread sheet and analysed using SPSS software. The frequency distribution of the demographic variables was obtained. The proportions and confidence limits were obtained for various antibiotics for susceptibility and resistance. The prevalence of P.aeruginosa and A.baumannii were obtained and data summarised using graphs and tables.

\section{Ethical considerations}

Waiver of informed consent was obtained with approval from the institutional ethics committee as only samples will be used in this study. All data will be stored using code numbers to conceal patient identity.

The study flow diagram using Strobe guidelines is given in Figure 1.

\section{Results and Discussion}

During the period of study from April 2017 to June 2017, a total of 83 endotracheal secretions from patients of different age groups admitted in the various intensive care units of the institution were processed. Non fermenters constituted $60.24 \%$ of the isolates. $P$. aeruginosa was the most commonly isolated non fermenter (54\%) followed by A.baumannii (30\%). Both these organisms were most frequently isolated from the Neuro Apex Care Unit. They were found to have a higher predilection for males. P.aeruginosa was most commonly isolated from individuals aged less than 50 years (38.9\%) while A.baumannii was most commonly isolated from individuals aged more than 50 years $(20 \%)$.
The mechanisms for antibiotic resistance amongst these organisms are diverse. Less than $15 \%$ P.aeruginosa were resistant to the 3rd and 4th generation cephalosporins, aminoglycosides and colistin while over $20 \%$ showed resistance to carbapenems and ciprofloxacin.

In the case of A.baumanii, $60-70 \%$ were resistant to carbapenems, 3rd and 4th generation cephalosporins and ciprofloxacin and over $60 \%$ to aminoglycosides. The resistance patterns for P.aeruginosa and A.baumannii for the selected antibiotics are given in Table 1.

The resistance patterns obtained were classified as multidrug resistance, extensive drug resistance and pan drug resistance based on accepted definitions and illustrated in Figure 2. Carbapenem resistance was found to be due to impermeability of carbapenems and expression of carbapenemase enzyme (metallo or oxa) as per Vitek reports.

\section{Legend}

Carbapenem resistance was found in over 70\%A.baumannii and over 20\% P.aeruginosa. $73 \%$ of A.baumannii were multi drug resistant and $60 \%$ were extensively drug resistant though there was no pan drug resistance. In the case of $P$. aeruginosa, over $10 \%$ were multi drug and extensively drug resistant and 3\% were pan drug resistant.

Our study documents the isolation of $60.24 \%$ non-fermenting gram negative bacilli with P.aeruginosa forming 54\% and A.baumannii forming $30 \%$ of the isolates. This is consistent with the results of Gladstone et al., ${ }^{11}$ and Nautiyal et al. ${ }^{12}$ 
Fig.1 Study flow diagram

Permission obtained from medical superintendent and approval obtained from Institutional Ethics Committee with waiver of informed consent.

\section{RECRUITMENT}

All endotracheal secretions coming to the microbiology department for culture and sensitivity during the study period $(\mathrm{n}=110)$

\section{ISOLATION}

Duplicate samples excluded $=27$

Samples processed using standard microbiological procedures $(n=83)$

\section{IDENTIFICATION}

Samples that yielded no growth $=5$.

Samples that yielded normal pharyngeal flora $=14$.

Samples that yielded other pathogenic organisms $=22$.

Samples that yielded $P$ sendomonas aeruginosa $(\mathrm{n}=27)$

ANTIBIOTIC SUSCEPTIBILITY

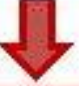

Antibiotic susceptibility NOT done $=0$

Antibiotic susceptibility testing done for Pseudomonas aeruginosa using Kirby Bauer disc diffusion method and automated analyser $(n=27)$
Samples that yielded Acinetobacter baumannii $(\mathrm{n}=15)$
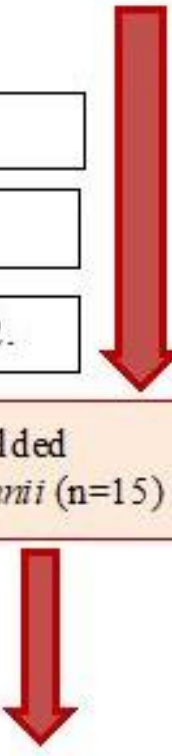

Antibiotic susceptibility testing done for Acinetobacter baumanni using Kirby Bauer disc diffusion method and automated analyser $(\mathrm{n}=15)$

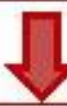

Data NOT analysed $(\mathrm{n}=0)$

Data analysed using SPSS statistical software. Frequencies and percentages and confidence limits were obtained.

$(\mathrm{n}=83)$

*The study flow chart in line with the STROBE (Strengthening the Reporting of Observational Studies in Epidemiology) statement (http://www.strobestatement.org). 
Table.1 Antibiotic resistance pattern of Pseudomonas aeruginosa and Acinetobacter baumannii

\begin{tabular}{|l|c|c|}
\hline \multicolumn{1}{|c|}{ Antibiotics } & $\begin{array}{c}\text { Pseudomonas aeruginosa } \\
(\boldsymbol{\%})\end{array}$ & $\begin{array}{c}\text { Acinetobacter baumannii } \\
(\boldsymbol{\%})\end{array}$ \\
\hline Ceftazidime & $14.81 \%$ & NA \\
\hline Cefoperazone/Sulbactam & $14.81 \%$ & $73.33 \%$ \\
\hline Doripenem & $22.22 \%$ & $73.33 \%$ \\
\hline Imipenem & $22.22 \%$ & $73.33 \%$ \\
\hline Meropenem & $22.22 \%$ & $73.33 \%$ \\
\hline Ciprofloxacin & $25.92 \%$ & $73.33 \%$ \\
\hline Amikacin & $11.11 \%$ & $60 \%$ \\
\hline Gentamicin & $11.11 \%$ & $60 \%$ \\
\hline Colistin & $14.81 \%$ & $0 \%$ \\
\hline Cefotaxime & NA & $80 \%$ \\
\hline Ceftriaxone & NA & $80 \%$ \\
\hline Tigecycline & NA & $0 \%$ \\
\hline
\end{tabular}

Fig.2 Classification of antibiotic resistance in Pseudomonas aeruginosa and Acinetobacter baumannii

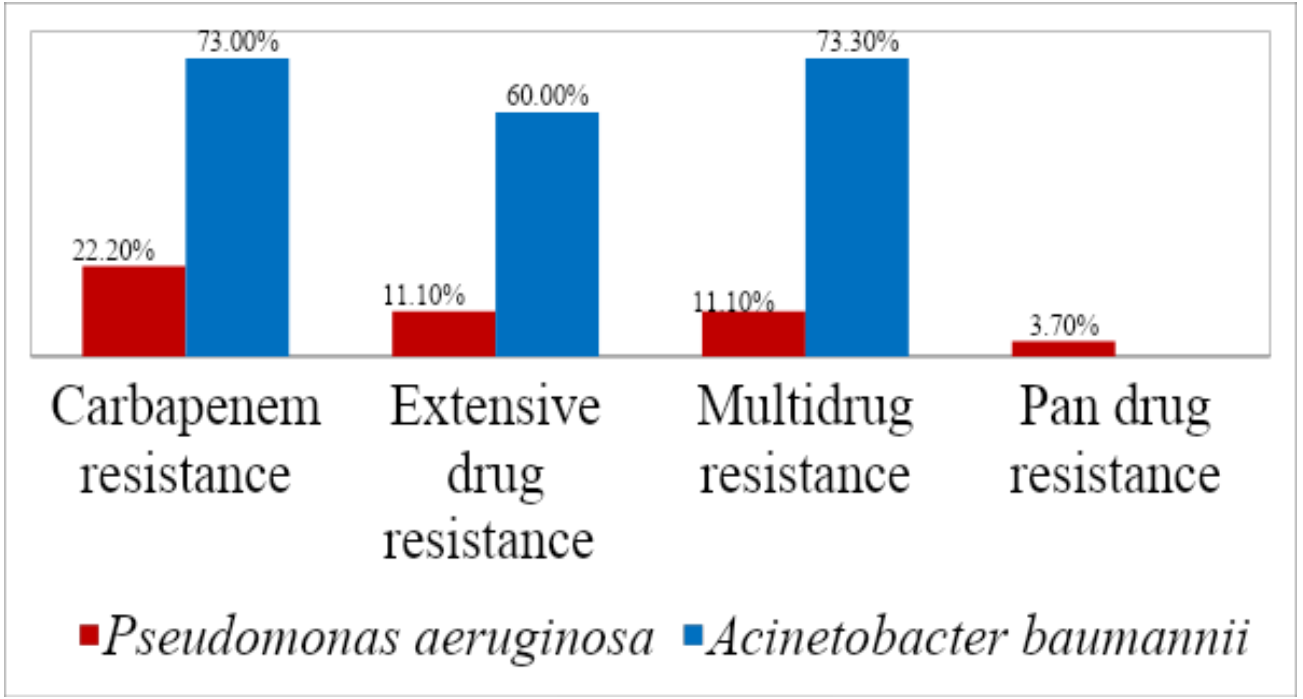

Indiscriminate use of antibiotics has led to a rise in resistance against the commonly used antibiotics. In our study $P$. aeruginosa shows the highest resistance to ciprofloxacin and the least resistance to amikacin and gentamicin. Acinetobacter baumannii remained highly resistant to most of the antibiotics tested except Colistin and Tigecycline.

The resistance could be attributed to decreased outer membrane permeability, increased efflux systems, alteration of penicillin binding proteins and expression of hydrolytic enzymes. The resistance could be chromosome or plasmid mediated and hence poses a serious risk for the spread of resistance by gene transfer among gram negative organisms ${ }^{[5]}$.

For many years, carbapenems have remained the drug of choice in the management of 
critically ill patients. But recently, resistance has started emerging rampantly against this useful group of antibiotics mainly because clinicians fail to step down to a narrower class of antibiotics once culture reports arrive. $\mathrm{CRAB}$ is a rising threat in many parts of the world. The emergence of CRAB is attributed to the horizontal spread of OXA type of Carbapenemase genes.

In our study, aminoglycosides were found to have good efficacy against P.aeruginosa. But its use is limited because it is rarely used as monotherapy, has poor penetration into lung and the central nervous system and also causes significant nephrotoxicity, ototoxicity and neuromuscular blockade.

No resistance has been reported in our study by Acinetobacter baumannii against colistin and tigecycline. Colistin is a useful polymyxin in multidrug resistant infections which has limited use owing to its properties of nephrotoxicity and neurotoxicity. Colistin monotherapy also bears the risk of producing hetero-resistance among gram negative populations exposed to colistin alone ${ }^{[16]}$.

Tigecycline on the other hand is a minocycline derivative with enhanced in vitro activity against gram positive and gram negative bacteria. Breakthrough bacteremia by Acinetobacter baumannii in patients receiving Tigecycline has been reported ${ }^{[14]}$. Also, Tigecycline resistance may emerge while the patient is on treatment [15]. Tigecycline has a pharmacokinetic profile not favourable for severe blood stream infections. Serum levels are low because of large distribution volume. This property allows it to be useful in surgical site infections not blood stream infections.

Combination therapy may help prevent emergence of resistance during treatment. According to studies, tigecycline and colistin combination regimen showed a significant decrease in bacterial load at 48 hours leading to a conclusion that together they may be used until there is an improvement of clinical signs, then treatment can be continued with monotherapy keeping in mind cost and side effects ${ }^{[17]}$.

In conclusion non fermenters constituted $60.24 \%$ of the 83 isolates obtained from endotracheal secretions of patients in this hospital. $P$. aeruginosa was the most commonly isolated non- fermenter (54\%) followed by A. baumannii (30\%).

In our study, aminoglycosides like amikacin and gentamicin were found to have good efficacy against $P$. aeruginosa. A. baumannii remained highly resistant to most of the antibiotics tested except colistin and tigecycline.

\section{Implications}

Appropriate choice of antibiotics while instituting empirical therapy is an important step in controlling antibiotic resistance. Periodic surveillance must be conducted in hospitals especially in intensive care units to appraise clinicians of the current trends of susceptibility patterns of circulating pathogens and tackle problems related to nosocomial infections caused by drug resistant pathogens.

\section{Acknowledgements}

We are grateful to the Dean and Management of the MOSC Medical college and the Research Department for the support and encouragement received to undertake this research.

\section{References}

1. Ananthanarayan R, Panicker CKJ. Arti Kapil ed. Textbook of Microbiology.9th edition. Hyderabad. University Press 
Publications. 2013; Ch. 33: 315-6, 44: 397-8.

2. Rit K, Saha R. Multidrug-resistant Acinetobacter infection and their susceptibility patterns in a tertiary care hospital. Nigerian Medical Journal.2012 Jul -Sep; 53(3): 126-8. doi: 10.4103/03001652.104379. PMCID: PMC3531029

3. Coppadoro A, Bellani G, Bronco A, Roberto B, Lucchini A, Bramati S et al., Measurement of endotracheal tube secretions volume by microcomputed tomography (MicroCT) Scan: an experimental and clinical study. BMC anesthesiology. 2014; 14: 22. doi:10.1186/1471-2253-14-22

4. Ramphal R, J C Sadoff, Pyle M, Silipigni J D. Role of pili in the adherence of Pseudomonas aeruginosa to injured tracheal epithelium. American society for Microbiology. 1984 April; 44: 38-40.

5. Rice L B. Challenges in Identifying New Antimiccrobial Agents Effective for Treating Infections with Acinetobacter baumannii and Pseudomonas aeruginosa. Clinical Infectious Diseases. 2006; 43: S100-5.

6. $\mathrm{Wu}$ Tak-chiu. Carbapenem-resistant or Multidrug-resistant Acinetobacter baumannii - a Clinician's Perspective. The Hong Kong Medical Diary.2011 April; 16: 4.

7. Malini A, Deepa EK, Gokul BN, et al. Non fermenting gram-negative bacilli infections in a tertiary care hospital in Kolar, Karnataka. J Lab Physicians 2009; 1(2): 62-6.

8. Jayaprakash C, Ummer N. Identification of non-fermentative Gram-negative bacilli isolated from clinical specimens. J.Evid. Based Med. Healthc. 2016; 3(27), 1212-1215. DOI: 10.18410/jebmh/2016/280.

9. Clinical Laboratory Standards Institute
(CLSI) Guidelines. Performance standards for antimicrobial Disk Susceptibility Testing; Twenty-Fifth Informational Supplement - M100-S25. January 2015.

10. Sample size measured using nMaster Sample Size Calculation software, version 2 produced by Department of Biostatistics, Christian Medical College, Vellore 632 004. Tamil Nadu. India.

11. Gladstone P, Rajendran P, Brahmadathan KN. Incidence of Carbapenem Resistant Non-fermenting Gram Negative Bacilli from patients with respiratory infections in the Intensive Care Units. Indian Journal of Medical Microbiology. 2005; 23(3): 189-191.

12. S. Nautiyal, S Jauhari, N Goel, B S Mahawal Current Trend of Nonfermenting Gram Negative Bacilli in a tertiary care hospital in Dehradun, Uttarakhand. International Journal of Advanced Research. 2014; 2(2): 322328.

13. Clin Microbiol Infect. 2012 Mar; 18(3): 268-81.doi:10.1111/j.14690691.2011.03570.x.Epub 2011 Jul 27.

14. Peleg AY, Potoski BA, Rea R, Adams J, Sethi J, Capitano B, Husain S, Kwak EJ, Bhat SV, Paterson DL. Acinetobacter baumannii bloodstream infection while receiving Tigecycline: a cautionary report. J Antimicrob Chemother. 2007 Jan; 59(1): 128-31.

15. Chan JD, Graves JA, Deltit TH. Antimicrobial treatment and clinical outcomes of Carbapenem resistant Acinetobacter baumannii Ventilatorassociated pneumonia. J Intensive Care Med. 2010 Sep 25(6):343-348.

16. Hawley JS, Murray CK, Jorgensen JH. Development of Colistin dependent Acinetobacter baumannii- Acinetobacter calcoaceticus complex. Antimicrob Agents Ch 51, 4529-4530(2007). 
17. Tuon FF, Rocha JL, Merlini AB. Combined therapy of multidrug resistant Acinetobacter baumannii infection- is there evidence outside the laboratory? Journal of Medical Microbiology (2015), 64, 951-959.
18. UK Standards for Microbiology Investigations. Identification of Pseudomonas species and other NonGlucose Fermenters. Bacteriology identification ID: 17 . Issue 3 . Issue date 13.04.15

\section{How to cite this article:}

Seba Miriam David, Chithra Jayaprakash and Anna Mathew. 2020. Isolation, Identification and Antibiotic Susceptibility Testing of Pseudomonas aeruginosa and Acinetobacter baumannii from Endotracheal Secretions in a Tertiary Care Centre. Int.J.Curr.Microbiol.App.Sci. 9(02): 1566-1574. doi: https://doi.org/10.20546/ijcmas.2020.902.180 